\title{
Feasibility of continuous, catheter-directed thrombolysis using low-dose urokinase in combination with low molecular-weight heparin for acute iliofemoral venous thrombosis in patients at risk of bleeding
}

\author{
GUOPING CHEN, WANGYIN SHI, XU HE, WENSHENG LOU, LIANG CHEN and JIANPING GU
}

Department of Interventional Radiology, Nanjing First Hospital, Nanjing Medical University, Nanjing, Jiangsu 210006, P.R. China

Received May 11, 2015; Accepted November 11, 2016

DOI: $10.3892 /$ etm.2017.4023

\begin{abstract}
The present study aimed to examine the feasibility of catheter-directed thrombolysis (CDT) using continuous infusion of low-dose urokinase in combination with low molecular weight heparin (LMWH) for acute iliofemoral venous thrombosis. This retrospective analysis included patients with symptomatic acute iliofemoral venous thrombosis who received CDT using continuous infusion of low-dose urokinase in combination with LMWH within the past four years. Urokinase was administered at $1 \times 10^{4} \mathrm{U} / \mathrm{h}$ and $2 \times 10^{4} \mathrm{U} / \mathrm{h}$ in patients at high-risk and low-risk of bleeding, respectively. Measurements included urokinase dosage, duration, clinical outcomes and CDT-related complications. A total of 46 patients were included (high-risk, n=17; low-risk, n=29). In the high-risk patients, 64.7\% experienced dissolution of $\geq 50 \%$ thrombi after a median CDT duration of 8 days (range, 6-10 days) and median total urokinase dose of $1.92 \times 10^{6}$ units (range, $1.44-2.4 \times 10^{6}$ units). In the low-risk patients, $82.8 \%$ achieved dissolution of $\geq 50 \%$ thrombi after a median CDT duration of 7 days (range, 4-10 days) and a median total urokinase dose of $3.36 \times 10^{6}$ units (range, 1.92-4.80 $\times 10^{6}$ units). Remission of clinical symptoms after CDT was achieved in $15(88.2 \%)$ and 28 (96.6\%) cases in high-risk and low-risk patients, respectively. No treatment-associated pulmonary embolism or major bleeding was observed. Three $(6.5 \%)$ subjects (high-risk, $\mathrm{n}=1$; low-risk, $\mathrm{n}=2$ ) experienced minor bleeding. In conclusion, continuous infusion of low-dose urokinase via CDT in combination with LMWH is effective and safe for acute iliofemoral venous thrombosis in patients with one or more risk factor for bleeding.
\end{abstract}

Correspondence to: Dr Wanyin Shi or Dr Jianping Gu, Department of Interventional Radiology, Nanjing First Hospital, Nanjing Medical University, 68 Changle Road, Nanjing, Jiangsu 210006, P.R. China E-mail: shwy110@163.com

E-mail: gjp_nj@163.com

Key words: deep venous thrombosis, iliofemoral thrombosis, catheter-directed thrombolysis, urokinase

\section{Introduction}

Deep vein thrombosis (DVT) is a serious medical disorder, one of the most concerning clinical issues of which is its association with fatal pulmonary embolism (1). The first episode incidence has been reported to be between 60 and 180 per 100,000 population per year, with a significant mortality rate of up to $25 \%(1,2)$. The risk factors vary, and are largely dependent upon the conditions of the individual, such as age $>$ older age $>65$ years, obesity, hypertension, metabolic syndrome and cigarette smoking, or are secondary to malignant tumor, major surgery, immobilization, pregnancy, trauma, oral contraceptives, and acute medical illness, such as pneumonia or congestive heart failure $(3,4)$.

Catheter-directed thrombolysis (CDT) is widely used in patients with deep vein thrombosis (DVT). In patients with unilateral DVT without inferior vena cava involvement, urokinase is typically given for no more than $96 \mathrm{~h}$ at $1.2-1.8 \times 10^{5} \mathrm{U} / \mathrm{h}$ with unfractionated heparin (UH) $(1,2)$. CDT has been recommended for use in selected patients with acute proximal DVT (5), as it can facilitate early removal of the thrombus and reduce acute symptoms and post-thrombotic morbidity. Overnight CDT infusion with high-dose urokinase is associated with $11 \%$ major bleeding and $16 \%$ minor bleeding (5-12), particularly in patients with one or more risk factor for bleeding.

Low molecular weight heparin (LMWH) is safer than UH for DVT (13-15), and is regarded as the first choice for patients with contraindications to thrombolysis. Use of LMWH alone is unable to clear the clots completely and is associated with a high risk of developing postthrombotic syndrome, thus compromising the quality of life. Although CDT is preferable to the use of LMWH alone for patients with DVT, it remains controversial whether patients at high-risk of bleeding can benefit from using CDT.

In order to maximize the benefits and minimize the associated bleeding, a modified CDT (with continuous infusion of urokinase at low dose) in combination with systemic LMWH is now used in our institution since its introduction in January 2011 for patients with unilateral DVT and one or more risk factor of bleeding. The present study is a 
retrospective analysis of these cases, with the aim of identifying the optimal CDT protocol that may benefit a population with DVT at risk of bleeding that is presumably contraindicated for administration of high-dose thrombolytic agent.

\section{Materials and methods}

Subjects. Medical records of all 46 patients who presented with first-time symptomatic acute iliofemoral DVT between January 2011 and December 2014 were analyzed. The study population had a mean age of $60.04 \pm 10.75$ years (range, 33-79 years), and a female predominance of $56.52 \%$. Diagnosis was performed based on medical history, physical examination, Doppler ultrasound and/or lower extremity venography. Treatment was initiated within 14 days from symptom onset in all cases. No patient who underwent the procedure had a contraindication to thrombolysis or anticoagulation (1). Patients with symptomatic pulmonary embolism or thrombosis in the inferior vena cava prior to the treatment were not included in the data analysis.

The following data were extracted: Demographics; clinical features related to thrombotic events; DVT risk factors; degree of thrombolysis; duration of CDT, remission of symptoms; total urokinase dose; CDT-related complications; and secondary interventional therapy, including percutaneous transluminal angioplasty (PTA) and stenting.

Ethical approval. The present study was approved by the hospital Institutional Review Board. All procedures performed in studies involving human participants were in accordance with the ethical standards of the institutional and/or national research committee and with the 1964 Helsinki declaration and its later amendments or comparable ethical standards. Written informed consent was obtained from all individual participants included in the study.

Patient assessment prior to thrombolytic therapy. Risk of bleeding was evaluated prior to CDT. High-risk was defined as: Patients aged $\geq 75$ years, prothrombin time $\geq 3 \mathrm{sec}$ above the normal upper limit, or 2-4 weeks after surgical or gynecological procedures, severe trauma, including spinal injury or closed thoracic and/or abdominal injury, or gastrointestinal bleeding.

Thrombolytic therapy. All patients were admitted to our center and stayed in the inpatients' wards for daily monitoring. All subjects received inferior vena cava filters via the femoral vein in the healthy limb prior to CDT. Retrograde catheterization of the femoral vein in the healthy lower extremity or antegrade catheterization of the popliteal vein in the affected lower extremity was performed. A Uni* Fuse $4 \mathrm{~F}$ infusion catheter (AngioDynamics, Latham, NY, USA) was used, and the length of the lateral-hole segment for placing into the thrombus was decided on the basis of thrombus distribution. Urokinase (Livzon Pharmaceutical Group, Inc., Gongbei, China) was delivered at $1 \times 10^{4}$ and $2 \times 10^{4} \mathrm{U} / \mathrm{h}$ in high-risk and low-risk patients, respectively. Stock solutions of 2.5 or $5 \times 10^{5}$ units urokinase were diluted to a final volume of $500 \mathrm{ml}$ in $0.9 \%$ physiological saline and infused continuously at $20 \mathrm{ml} / \mathrm{h}$.
During CDT, LMWH (Hebei Changshan Biochemical Pharmaceutical, Shijiazhuang, China) was injected subcutaneously at a dose of $4.1 \times 10^{3}$ IU every $12 \mathrm{~h}$.

Thrombolysis monitoring. Venography was performed every 2-3 days. Outcomes were classified as complete thrombolysis $(\geq 99 \%)$, partial thrombolysis (50-99\%), and minor thrombolysis $(<50 \%)(5)$. Coagulation function was monitored every two days or more often if necessary, including the prothrombin time, international normalized ratio (INR), plasma fibrinogen (FIB) concentration, activated partial thromboplastin time and platelet count.

Urokinase dosage was reduced by $50 \%$ if plasma FIB concentration decreased to $<1.5 \mathrm{~g} / \mathrm{l}$; coagulation function was assayed daily in such patients. If plasma FIB concentration dropped to $<1 \mathrm{~g} / \mathrm{l}$, CDT was suspended until examination on the following day. If FIB remained at $<1 \mathrm{~g} / \mathrm{l}$, CDT was discontinued permanently.

CDT was discontinued upon major bleeding $(16,17)$. CDT was temporarily suspended upon minor bleeding $(1,17)$. If the minor bleeding was solved the following day, CDT was resumed at a reduced dosage (50\%); if the bleeding continued, CDT was discontinued permanently.

Upon complete thrombolysis, CDT was discontinued. Upon partial thrombolysis, CDT was continued and the catheter position was adjusted if considered necessary. The maximum duration of CDT was 10 days. Throughout CDT, patients were advised to rest in bed, and the affected limb was placed at an upward angle of $30^{\circ}$. Assisted ankle joint-toe movement was carried out.

Post-thrombolysis procedures. Following CDT, patients with vascular stenosis $(\geq 30 \%)$ or occlusion underwent PTA and/or stenting of the narrowed and occlusive vessels. Patients were advised to wear graded-compression stockings with an ankle pressure of 30-40 mmHg. LMWH therapy overlapped with oral warfarin therapy for 5 days. Warfarin dose was adjusted to maintain the INR at 2-3 for at least 6 months.

Thrombolysis outcomes. Efficacy outcome was thrombolysis degree after CDT. Immediate clinical improvement was defined by a decrease in pain and/or swelling of the affected extremity within $24 \mathrm{~h}$ of CDT. Remission was defined as a difference of thigh circumference $(20 \mathrm{~cm}$ from the patella upper margin) by $\leq 2 \mathrm{~cm}$ between the affected limb and the healthy limb.

Safety outcomes included CDT-related complications during hospital stay, including major and minor bleeding, symptomatic pulmonary embolism and death, catheter indwelling-induced infection, and secondary thrombosis.

\section{Results}

Demographics and clinical characteristics of patients with $D V T$. A total of 46 patients received low-dose urokinase CDT in combination with LMWH. Demographics, clinical characteristics and DVT risk factors are shown in Table I. As outlined, 17 patients had a high risk of bleeding (Table II) and the remaining 29 were low-risk. Retrograde and antegrade catheterization was performed in 16 and 30 patients, respec- 
Table I. Demographic, clinical characteristics and DVT risk factors of 46 patients with acute unilateral iliofemoral venous thrombosis.

\begin{tabular}{|c|c|c|}
\hline Characteristic & At high risk of bleeding $(\mathrm{n}=17)$ & At low risk of bleeding $(n=29)$ \\
\hline Age, years & $66.29 \pm 11.83$ & $58.76 \pm 9.81$ \\
\hline \multicolumn{3}{|l|}{ Gender, \% (n/N) } \\
\hline Male & $47.06(8 / 17)$ & $41.38(12 / 29)$ \\
\hline Female & $52.94(9 / 17)$ & $58.62(17 / 29)$ \\
\hline Body weight, kg & $66.35 \pm 8.97$ & $63.69 \pm 6.34$ \\
\hline Interval between symptom onset and admission, days & $7.76 \pm 3.23$ & $7.64 \pm 3.83$ \\
\hline \multicolumn{3}{|l|}{ Limb circumference, $\mathrm{cm}$} \\
\hline Mid-crus of the affected limb ${ }^{a}$ & $44.06 \pm 11.10$ & $46.43 \pm 3.43$ \\
\hline Mid-thigh of the affected limb $b^{b}$ & $57.62 \pm 5.45$ & $57.53 \pm 4.07$ \\
\hline \multicolumn{3}{|l|}{ Site of thrombus, $\%(\mathrm{n} / \mathrm{N})$} \\
\hline Left lower extremity & $70.59(12 / 17)$ & $65.52(19 / 29)$ \\
\hline Right lower extremity & $29.41(5 / 17)$ & $34.48(10 / 29)$ \\
\hline \multicolumn{3}{|l|}{ DVT risk factors $^{\mathrm{c}}, \mathrm{n}$} \\
\hline Hypercoagulable state ${ }^{\mathrm{d}}$ & 1 & 2 \\
\hline Surgery or gynecological procedure & 5 & 5 \\
\hline Trauma & 2 & 3 \\
\hline Tumor & 2 & 3 \\
\hline Restricted movement & 7 & 9 \\
\hline May-Thurner syndrome & 7 & 16 \\
\hline Unknown & 1 & 4 \\
\hline
\end{tabular}

Values are reported as mean \pm standard deviation, unless otherwise indicated. ${ }^{\mathrm{a}} 10 \mathrm{~cm}$ from lower margin of the tibial tuberosity; ${ }^{\mathrm{b}} 15 \mathrm{~cm}$ from upper margin of the patella. ${ }^{c}$ Multiple causes were assigned to some patients, and no cause was detected in some patients. ${ }^{\mathrm{d}}$ Documented biochemical hypercoagulable disorder, based on deficiency in natural protein $\mathrm{C}$ or protein $\mathrm{S}$ or a history of persistent positivity for anticardiolipin antibodies. DVT, deep vein thrombosis.

tively (Figs. 1 and 2). Inferior vena cava filters were placed using a TrapEase permanent filter $(n=5)$, recyclable OptEase filter (both Cordis Corp., Miami Lakes, FL, USA; n=3) or recyclable Aegisy filter (Lifetech Scientific, Shenzhen, China; $\mathrm{n}=38)$. The filter was removed from 37 patients $(90.2 \%$ in those receiving recyclable filters) after a median duration of 10 days (range, 4-12 days). Thrombus-like debris was observed in the filters of 6 patients upon removal. Recyclable filters were not removed from 4 patients with a risk of venous thrombus recurrence. For DVT intended for CDT treatment, caval filter placement was usually performed to prevent the migration of clot debris into pulmonary arteries.

Angiographic outcomes of CDT. Following CDT, venography of the lower extremity exhibited complete thrombolysis or dissolution of most thrombi (partial thrombolysis) in 28 patients: 7 in high-risk patients and 21 in low-risk patients. All 28 patients showed iliac vein stenosis or occlusion following CDT and were treated by PTA using a balloon dilatation catheter (Medtronic, Inc., Minneapolis, MN, USA) with a diameter of 8-12 $\mathrm{mm}$ and/or stenting using Luminexx self-expanding stents (C. R. Bard, Inc., Karlsruhe, Germany; n=22) or Protege GPS self-expanding peripheral stents (ev3, Plymouth, MN, USA; $\mathrm{n}=6$ ) with a diameter of 10-14 $\mathrm{mm}$.
Table II. High risk factor of bleeding of the 17 patients.

\begin{tabular}{|c|c|}
\hline High risk factor of bleeding & No. of patient \\
\hline$\geq 75$ years of age (range:76-83 years) & 6 \\
\hline Prothrombin time $\geq 3 \mathrm{sec}$ beyond the NUL & 1 \\
\hline Surgical procedures $^{\mathrm{a}}$ & 3 \\
\hline Gynecological procedures $^{\mathrm{a}}$ & 2 \\
\hline \multicolumn{2}{|l|}{ Severe trauma ${ }^{a}$} \\
\hline Spinal injury & 1 \\
\hline Closed thoracic injury & 1 \\
\hline Gastrointestinal bleeding $^{\mathrm{a}}$ & 3 \\
\hline
\end{tabular}

${ }^{\mathrm{a}}>2$ weeks but $<4$ weeks prior to catheter-directed thrombolysis. NUL, normal upper limit.

Dissolution of $\geq 50 \%$ thrombi was achieved in $64.7 \%$ of high-risk patients, including $5.9 \%$ complete thrombolysis and $58.8 \%$ partial thrombolysis. The remaining $35.3 \%$ of patients achieved dissolution of $<50 \%$ thrombi. CDT was continued for a median of 8 days (range, 6-10 days); the median urokinase dose was $1.92 \times 10^{6}$ units (range, $1.44-2.4 \times 10^{6}$ units). 
A

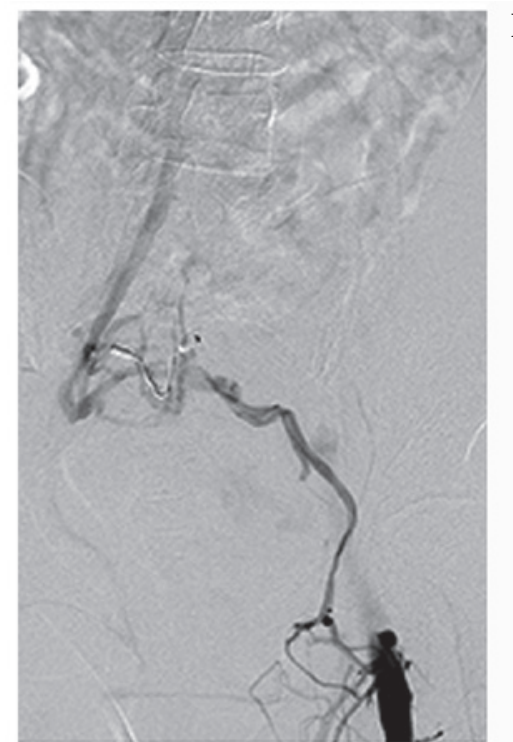

B

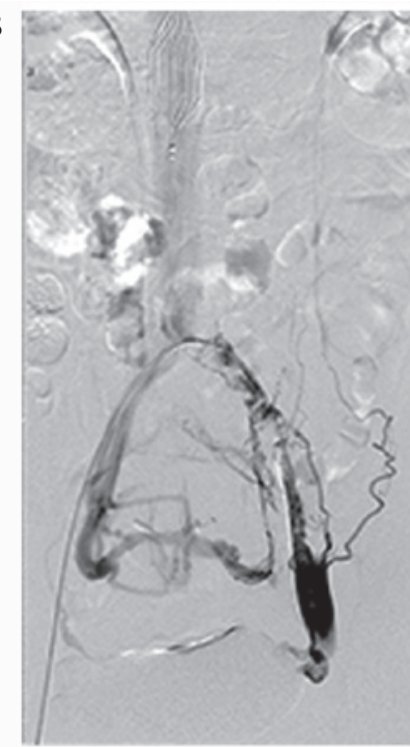

C

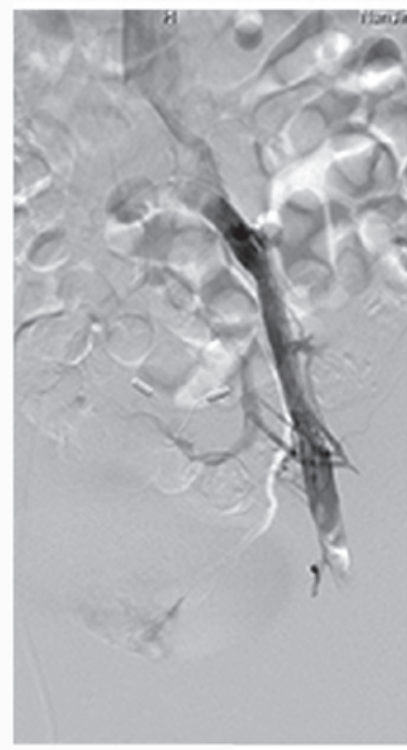

D

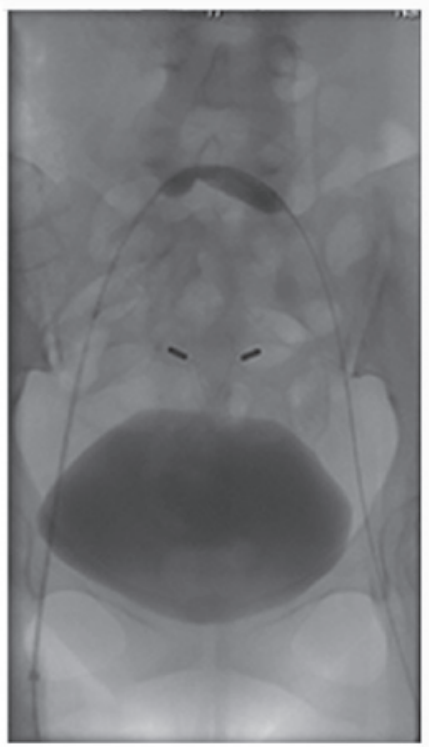

$\mathbf{E}$

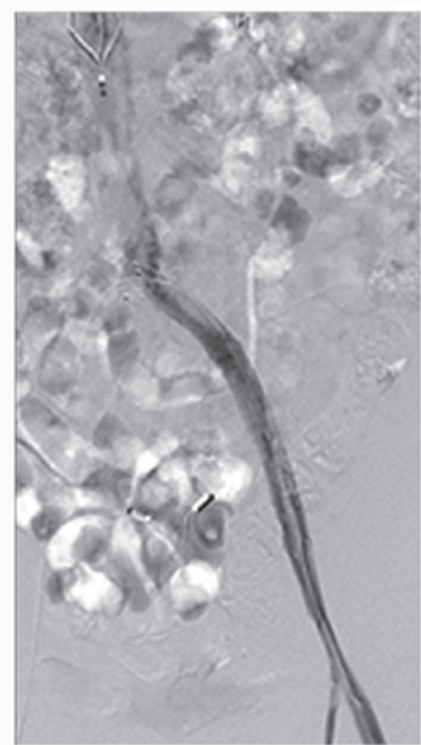

Figure 1. Angiography of a 45-year-old female patient with complaints of 3-day swelling and pain in the left lower extremity. (A) Antegrade angiography of the superficial dorsal vein of the left lower extremity reveals obstruction of blood flow regurgitation at the common femoral vein. Blood flows into the contralateral iliac vein via collateral vessels, and is subsequently regurgitated into the inferior cava vena. (B) Angiography of retrograde catheterization with a $4 \mathrm{~F}$ Uni" Fuse infusion catheter after placement of an inferior vena cava filter via the femoral vein in the healthy lower extremity. Imaging shows numerous thrombus-like shadows in the lumen of the left external iliac vein and common iliac vein. Pelvic collateral vessels are visible. (C) Angiography of retrograde catheterization with a $4 \mathrm{~F}$ Uni" Fuse infusion catheter after 6 days of catheter-directed thrombolysis with urokinase at a dose of $2 \times 10^{4} \mathrm{U} / \mathrm{h}$. No evidence of thrombus is visible in the lumen of the left external iliac vein or common iliac vein. The lumen of the left common iliac vein appears to narrow before connecting with the inferior vena cava, and fewer collateral vessels than before thrombolysis are visible. (D) Balloon dilatation $(10 \times 40 \mathrm{~mm})$ in the left common iliac vein. The balloon takes on a constrict trace appearance, reflecting severe stenosis. (E) Angiography of retrograde catheterization with a $\mathrm{H} 1$ catheter following implantation of a stent $(12 \times 80 \mathrm{~mm})$ in the left common iliac vein and external iliac vein. Imaging shows unobstructed blood flow in the left common femoral vein, external iliac vein and common iliac vein, as well as in the stent. No collateral vessels are visible.

Notably, $82.8 \%$ of the low-risk patients achieved dissolution of $\geq 50 \%$ thrombi, including $27.6 \%$ complete thrombolysis and $55.2 \%$ partial thrombolysis. The remaining $17.2 \%$ exhibited dissolution of $<50 \%$ thrombi. CDT lasted for a median of 7 days (range, 4-10 days); the median urokinase dose was $3.36 \times 10^{6}$ units (range, $1.92-4.80 \times 10^{6}$ units). For patients with acute proximal DVT, continuous CDT using low-dose urokinase offered favorable angiographic outcomes both in low-risk and high-risk groups

Clinical outcomes and CDT-related complications. Immediate clinical improvement was observed in $14(82.3 \%)$ and $27(93.1 \%)$ cases in high-risk and low-risk patients, respectively. Remission of clinical symptoms after CDT was achieved in $15(88.2 \%)$ and $28(96.6 \%)$ cases in high risk and low-risk patients, respectively. Symptomatic pulmonary embolism or major bleeding was not observed. Three subjects (high-risk group, $\mathrm{n}=1$; low-risk group, $\mathrm{n}=2$ ) experienced minor bleeding. The rate of minor bleeding was $6.5 \%$. The minor bleeding event in the high-risk patients was hematuresis after 6 days of CDT $(n=1)$. Bleeding events in the low-risk patients included hematuresis after four days of $\mathrm{CDT}(\mathrm{n}=1)$ and errhysis at the margin of the vagina vasorum after five days of CDT $(n=1)$. CDT was discontinued permanently in the two patients who experienced hematuresis. 


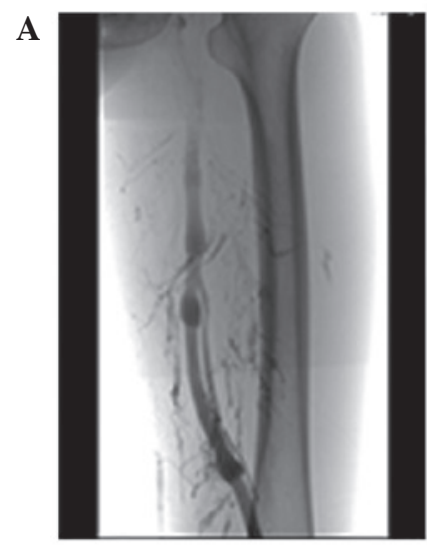

$\mathbf{E}$

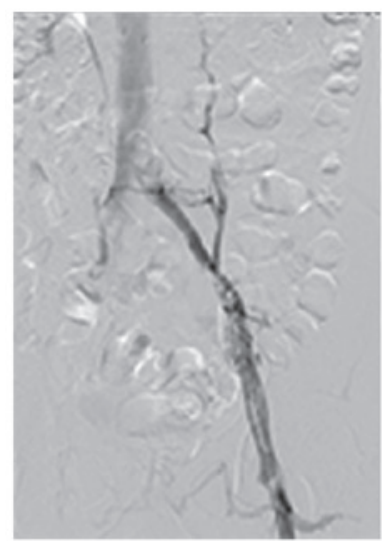

B

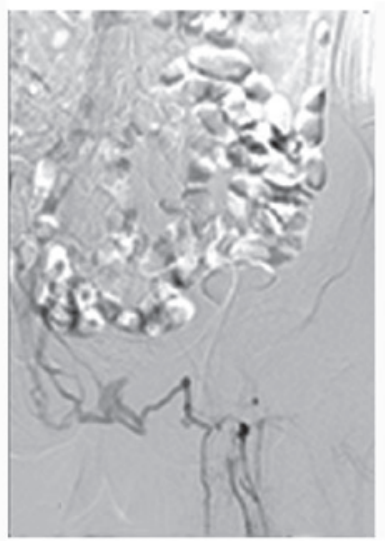

F

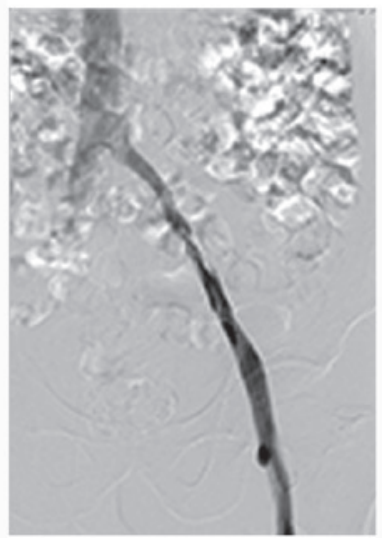

C

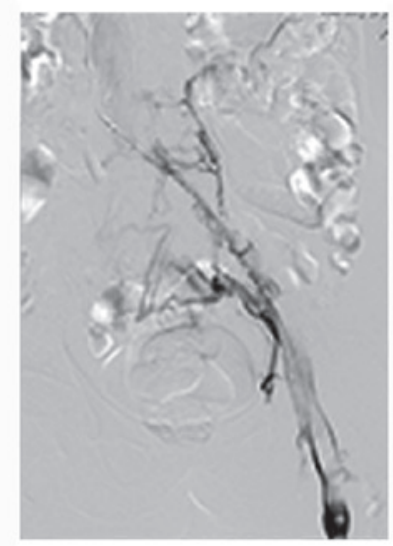

G

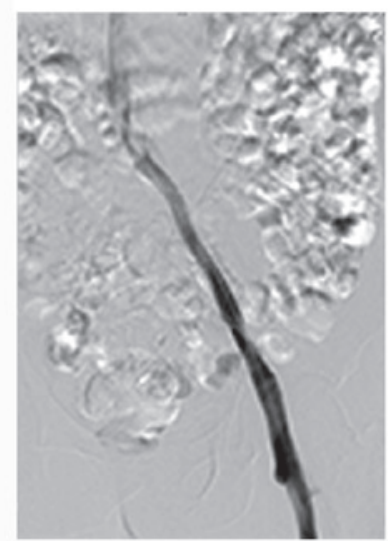

D

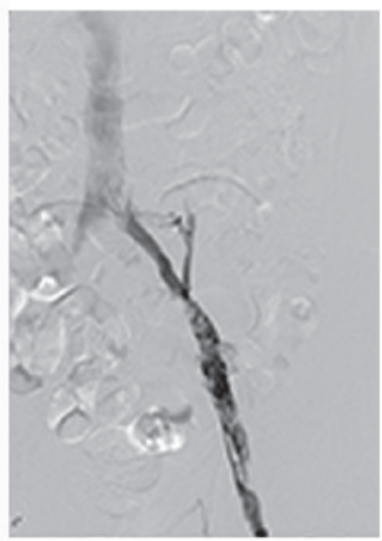

H

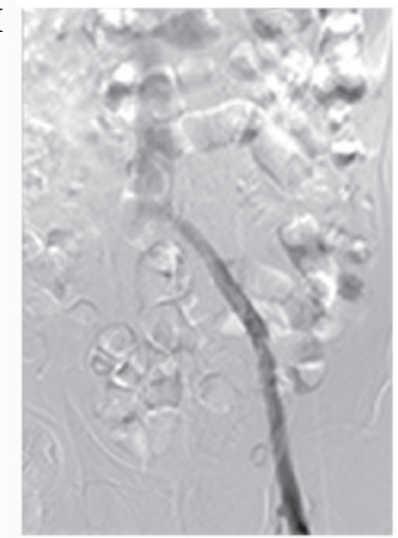

Figure 2. Angiography of a 68-year-old man with complaints of 5-day swelling and pain in the left lower extremity. (A) Antegrade angiography of the superficial dorsal vein of the left lower extremity shows the unobstructed lumen in the middle and lower segments of the left superficial femoral vein, obstruction due to contrast agent reflux in the upper segment of the left superficial femoral vein, and some tributaries of the deep femoral vein and collateral vessels. (B) Imaging showing contrast agent entering the inferior cava vena after affluxing into the contralateral iliac vein via collateral vessels in the pelvic wall and perineum. The main trunk of the upper segment of the left superficial femoral vein, the common femoral vein, the external iliac vein and the common iliac vein are not visible. (C) Angiography of antegrade catheterization with a 4F Uni"Fuse infusion catheter inserted in the popliteal vein in the affected lower extremity. Images were captured with the patient in a supine position following implantation of an Aegisy inferior vena cava filter via the right femoral vein under local anesthesia. Imaging shows numerous filling defects in the lumen of the upper segment of the left superior femoral vein, common femoral vein, external iliac vein and common iliac vein. Pelvic collateral vessels are visible. (D) Repeat angiography after two days of catheter-directed thrombolysis with urokinase at a dose of $2 \times 10^{4} \mathrm{U} / \mathrm{h}$ reveals remarkable reduction in filling defects in the lumen of the upper segment of the left superior femoral vein, common femoral vein, external iliac vein and common iliac vein. The lumen of the left common iliac vein appears to narrow prior to afflux into the inferior vena cava, and fewer collateral vessels than before thrombolysis are visible. (E) Image obtained after positioning the tip of the 4F Uni"Fuse infusion catheter into the left external iliac vein following the capture of panel D. The results here are similar to those in panel D. (F) Repeat angiography after four days of catheter-directed thrombolysis with urokinase at a dose of $2 \times 10^{4} \mathrm{U} / \mathrm{h}$ reveals few filling defects in the lumen of the left external iliac vein as well as a narrow lumen. (G) Angiography after removal of the inferior vena cava filter and implantation of a stent $(10 \times 10 \mathrm{~mm})$ via the popliteal vein shows unobstructed blood flow in the left iliac venous stent and inferior cava vena. Imaging also shows an intact vascular wall. (H) Antegrade angiography of the superficial dorsal vein of the left lower extremity at discharge from hospital reveals unobstructed blood flow in the main trunk of the femoral vein and iliac vein, as well as afflux of contrast agent into the inferior cava vena.

Five patients (10.9\%) experienced a drop in plasma FIB concentration to $<1 \mathrm{~g} / 1$ after five days of CDT, among which two were high-risk patients. CDT was temporarily suspended due to a decrease of plasma FIB in three cases, and discontinued permanently in two cases. None of the five patients received plasma transfusion. No thrombi developed as a result of long-term catheter indwelling. The outcomes of thrombolysis in 46 patients are shown in Table III. The duration of CDT and number of patients undergoing CDT are shown in Fig. 3. The findings indicated that continuous CDT using low-dose urokinase was technically feasible and safe for patients with DVT, even those at risk of bleeding.

\section{Discussion}

CDT is the first choice for the treatment of symptomatic iliofemoral venous thrombosis $(1,18,19)$. However, there is no consensus on whether particular doses or administration methods for thrombolytics (intermittent, continuous or a mixture) are more effective than others. Furthermore, CTD, particularly short-duration, high-dose thrombolytic therapy, is associated with a substantial risk of bleeding. In overnight CDT with high-dose urokinase, urokinase is administered at a loading dose followed by a maintenance dose of $0.8-2.4 \times 10^{5} \mathrm{U} / \mathrm{h}$. During thrombolysis, systemic UH is administered at a therapeutic dose or sub-therapeutic dose (250-500 U/h) until aPTT is 70-90 sec or higher and the aPTT ratio reaches 1.5-3.0. The coagulation index is reexamined every 4-6 h, and venography is performed every 6-24 h. If thrombolysis is determined to be ineffective by two successive venography examinations or upon 96-h thrombolysis, CDT is abandoned, with a final mean total urokinase dose at $1.98-7.8 \times 10^{6} \mathrm{U}(5-12)$. Despite frequent monitoring, bleeding events could not be avoided $(6,10,15)$. 
Table III. Degree of thrombolysis, median duration of CDT, median total urokinase dose, clinical outcomes and CDT-related complications of 46 patients.

\begin{tabular}{lcc}
\hline Characteristics & High-risk patients $(\mathrm{n}=17)$ & Low-risk patients $(\mathrm{n}=29)$ \\
\hline Degree of thrombolysis, $\mathrm{n}(\%)$ & & \\
Complete $(\geq 99 \%)$ & $1(5.9)$ & $8(27.6)$ \\
Most $(50-99 \%)$ & $10(58.8)$ & $16(55.2)$ \\
Minor $(<50 \%)$ & $6(35.3)$ & $8(17.2)$ \\
Duration of CDT (median, U) & 8 & 7 \\
Total urokinase dose (median, $\mathrm{U})$ & $1.92 \times 10^{6}$ & $3.36 \times 10^{6}$ \\
Clinical outcomes, $\mathrm{n}(\%)$ & & \\
Immediate clinical improvement & $14(82.3)$ & $27(93.1)$ \\
Remission of clinical symptoms & $15(88.2)$ & $28(96.6)$ \\
Complications, $\mathrm{n}(\%)$ & & \\
Major bleeding & 0 & 0 \\
Minor bleeding & $1^{\mathrm{a}}(5.9)$ & $2^{\mathrm{b}}(6.9)$ \\
FIB drop & $2(1.8)$ & $3(0.3)$
\end{tabular}

${ }^{\mathrm{a} H e m a t u r e s i s}$; ${ }^{b}$ hematuresis $(\mathrm{n}=1)$ and errhysis $(\mathrm{n}=1)$ at the margin of the vagina vasorum. CDT, catheter-directed thrombolysis; FIB, fibrinogen.

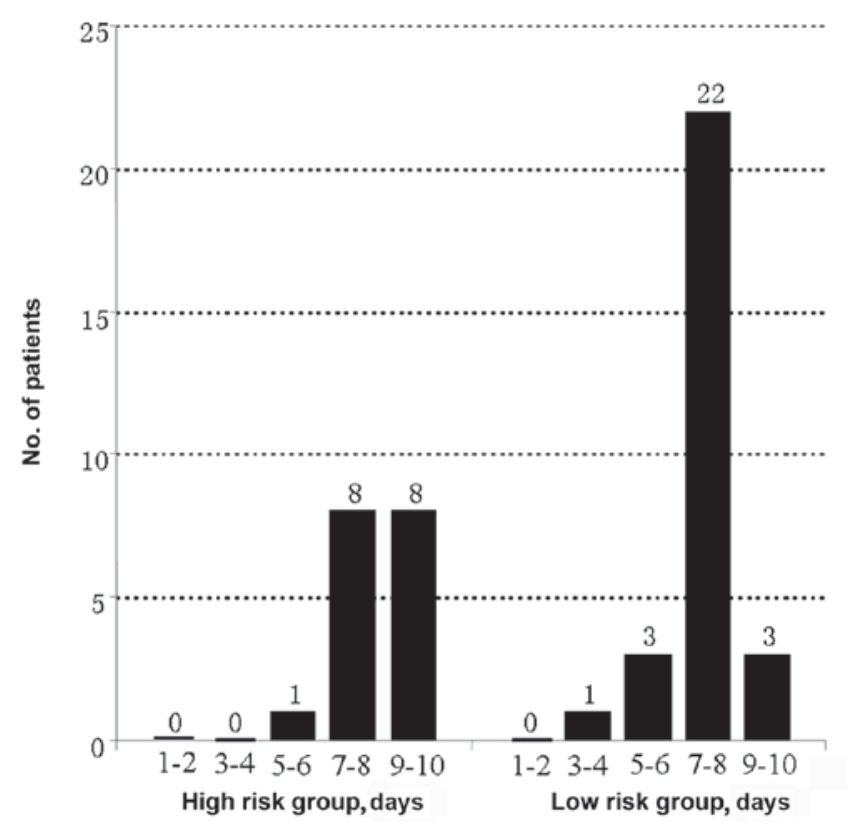

Figure 3. Duration of catheter-directed thrombolysis in patients with acute unilateral iliofemoral venous thrombosis, depending on whether the patients were categorized as at low or high risk of bleeding.

A multicenter prospective trial (5) has previously reported using urokinase with a mean infusion time of $53.4 \mathrm{~h}$ (range, 2-147.3 h) and a mean urokinase dose of $7.8 \times 10^{6}$ units (range, $0.5-4.4 \times 10^{7}$ units), corresponding to a mean urokinase infusion rate of $1.46 \times 10^{5} \mathrm{U} / \mathrm{h}$. Numerous studies have reported a comparable mean urokinase infusion rate of $0.8-2.4 \times 10^{5} \mathrm{U} / \mathrm{h}(7,10-12)$. The multicenter study achieved $31 \%$ complete thrombolysis; $52 \%$ dissolution of most thrombi (partial thrombolysis), and a $17 \%$ dissolution of $<50 \%$ thrombi (5). In the present study, urokinase infu- sion at a rate of only $1 \times 10^{4} \mathrm{U} / \mathrm{h}$ in high-risk patients yielded $64.7 \%$ dissolution of $\geq 50 \%$ thrombi. Urokinase infusion at a rate of only $2 \times 10^{4} \mathrm{U} / \mathrm{h}$ in low-risk patients yielded $82.8 \%$ dissolution of $\geq 50 \%$ thrombi. The present results in low-risk patients are similar to those of the multicenter study ( $83 \%$ dissolution of $\geq 50 \%$ thrombi), whereas only half dosage was required (3.36 vs. $7.8 \times 10^{6} \mathrm{U}$ ). In general, the efficacy (dissolution of $\geq 50 \%$ thrombi) of our approach is comparable to that reported with high-dose, short-term CDT (80-90\% dissolution of $\geq 50 \%$ thrombi) $(5,7,9,10,12)$.

In the present study, the rate of dissolution of $\geq 50 \%$ thrombi was lower in high-risk than in low-risk patients (64.7 vs. 82.8\%). The rate of complete thrombolysis was also lower in high-risk patients (5.9 vs. $27.6 \%$ ), suggesting that the infusion rate of urokinase must reach a critical level for thrombus dissolution.

UH in conventional therapy may also increase the risk of bleeding. Nazir et al (18) argued that coagulation must recover to normal (INR at 2-3) prior to thrombolysis in order to reduce the risk of bleeding. The introduction of subcutaneously administered LMWH offers significant advantages over UH since LMWH induces less hemorrhagic complications than UH (15). The present findings of no major bleeding and $6.5 \%$ minor bleeding are comparable to studies of CDT (no major bleeding and 4.8-13\% minor bleeding) involving urokinase doses of $1.25-7.8 \times 10^{4} \mathrm{U} / \mathrm{h}$ and total doses $\leq 3.5 \times 10^{6} \mathrm{U}(20-22)$. The dosage of LMWH at $4.1 \times 10^{3} \mathrm{IU}$ every $12 \mathrm{~h}$ seemed to be appropriate since we did not observe thrombus expansion or secondary thrombus development in any of the cases.

The CDT duration in the present study was considerably longer than in the multicenter study (168 vs. 53.4 h) (5). The three minor bleeding events in our study all occurred after four days of CDT, which is consistent with increased incidence of bleeding events with increasing CDT infusion duration (23). However, the CaVenT trial (24) did not conclude that there 
was a higher risk of hemorrhagic events upon prolonged CDT. Several other studies also failed to demonstrated a higher risk of major bleeding events upon extended infusion duration (20-22). Nevertheless, the Society of Interventional Radiology recommends $96 \mathrm{~h}$ as the maximum duration of thrombolytic therapy involving urokinase infusion, in order to reduce the occurrence of bleeding events (1). The use of higher doses of thrombolytic agent and heparin is likely to accelerate and increase lysis and reduce treatment time; however, it may be associated with increased bleeding risks. To reduce the hemorrhagic risks and thrombolytic infusion times, percutaneous mechanical thrombectomy has emerged as an option for the treatment of acute DVT $(12,16,18,23)$.

CDT is suitable for patients with low-risk bleeding $(1,18,19)$; however, defining subjects as at low risk of bleeding may be difficult due to bleeding during thrombolytic therapy. Therefore, all patients undergoing thrombolytic therapy require an assessment of the factors that may increase the risk of bleeding. Prior to thrombolytic therapy in the present study, subjects were stratified into high- and low-risk bleeding groups based on risk factors, and distinct rates of urokinase infusion were used. Patients older than 75 years and patients who experienced recent major or serious surgery, trauma or gastrointestinal bleeding are typically excluded from CDT therapy $(5,16,22,25)$. The present study included six senile patients (aged $>75$ years) receiving $1 \times 10^{4} \mathrm{U} / \mathrm{h}$ urokinase with no bleeding events (either major or minor). We believe that a robust screening of the risk factors helped to mitigate the risk of bleeding in the present study.

One of the limitations of the protocol used in the present study is the relatively long dwelling duration of the catheter, which may led to discomfort that was not tolerated by some patients and was associated with some potential complications, such as catheter-related infection and catheter migration. Based on our experiences, assisted ankle joint-toe movement may improve patients' comfort and compliance.

No instances of symptomatic pulmonary embolism were observed, likely reflecting the use of inferior vena cava filters; indeed, thrombus-like debris was found in 6/37 filters removed from patients after CDT. Whether such filters prevent pulmonary embolism during CDT remains controversial (5,25-27). Consistent with the findings of previous studies $(1,7,11,28)$, all patients in our study who experienced dissolution of all or the majority of venous thrombi required PTA and stenting after CDT in order to regain forward blood flow in the main trunk of the iliofemoral vein.

In conclusion, the present findings suggest that continuous infusion of low-dose urokinase via CDT in combination with LMWH is effective and safe for treating acute iliofemoral venous thrombosis, particularly in patients with at least one risk factor for bleeding

\section{Acknowledgements}

This study was supported by grants from the Special Scientific Research in Clinical Medicine Program of the Jiangsu Provincial Science and Technology Development Foundation (grant no. BL2014013), from the Key Program of the Nanjing Medical Science and Technology Development Foundation (grant no. ZKX10004) and from the 12th Five-Year Plan of the Nanjing Medical Science and Technology Development Foundation (grant no. ZDK12016).

\section{References}

1. Vedantham S, Sista AK, Klein SJ, Nayak L, Razavi MK, Kalva SP, Saad WE, Dariushnia SR, Caplin DM, Chao CP, et al; Society of Interventional Radiology and Cardiovascular and Interventional Radiological Society of Europe Standards of Practice Committees: Quality improvement guidelines for the treatment of lower extremity deep vein thrombosis with use of endovascular thrombus removal. J Vasc Interv Radiol 25: 1317-1325, 2014.

2. Jaff MR, McMurtry MS, Archer SL, Cushman M, Goldenberg N, Goldhaber SZ, Jenkins JS, Kline JA, Michaels AD, Thistlethwaite P, et al; American Heart Association Council on Cardiopulmonary, Critical Care, Perioperative and Resuscitation; American Heart Association Council on Peripheral Vascular Disease; American Heart Association Council on Arteriosclerosis, Thrombosis and Vascular Biology: Management of massive and submassive pulmonary embolism, iliofemoral deep vein thrombosis, and chronic thromboembolic pulmonary hypertension: A scientific statement from the American Heart Association. Circulation 123: 1788-1830, 2011.

3. Goldhaber SZ, and Bounameaux H: Pulmonary embolism and deep vein thrombosis. Lancet 379: 1835-1846, 2012.

4. Wong $\mathrm{P}$, and Baglin T: Epidemiology, risk factors and sequelae of venous thromboembolism. Phlebology 27 (Suppl 2): 2-11, 2012.

5. Mewissen MW, Seabrook GR, Meissner MH, Cynamon J, Labropoulos N and Haughton SH: Catheter-directed thrombolysis for lower extremity deep venous thrombosis: Report of a national multicenter registry. Radiology 211: 39-49, 1999.

6. Grunwald MR and Hofmann LV: Comparison of urokinase, alteplase, and reteplase for catheter-directed thrombolysis of deep venous thrombosis. J Vasc Interv Radiol 15: 347-352, 2004.

7. Kwak HS, Han YM, Lee YS, Jin GY and Chung GH: Stents in common iliac vein obstruction with acute ipsilateral deep venous thrombosis: Early and late results. J Vasc Interv Radiol 16: 815-822, 2005.

8. Janssen MC, Wollersheim H, Schultze-Kool LJ, and Thien T: Local and systemic thrombolytic therapy for acute deep venous thrombosis. Neth J Med 63: 81-90, 2005.

9. Kim HS, Preece SR, Black JH, Pham LD and Streiff MB: Safety of catheter-directed thrombolysis for deep venous thrombosis in cancer patients. J Vasc Surg 47: 388-394, 2008.

10. Manninen H, Juutilainen A, Kaukanen E, and Lehto S: Catheter-directed thrombolysis of proximal lower extremity deep vein thrombosis: A prospective trial with venographic and clinical follow-up. Eur J Radiol 81: 1197-1202, 2012.

11. Semba CP and Dake MD: Iliofemoral deep venous thrombosis: Aggressive therapy with catheter-directed thrombolysis. Radiology 191: 487-494, 1994.

12. Kim HS, Patra A, Paxton BE, Khan J and Streiff MB: Adjunctive percutaneous mechanical thrombectomy for lower-extremity deep vein thrombosis: Clinical and economic outcomes. J Vasc Interv Radiol 17: 1099-1104, 2006.

13. Cossette B, Pelletier MÈ, Carrier N, Turgeon M, Leclair C, Charron P, Echenberg D, Fayad T and Farand P: Evaluation of bleeding risk in patients exposed to therapeutic unfractionated or low-molecular-weight heparin: A cohort study in the context of a quality improvement initiative. Ann Pharmacother 44: 994-1002, 2010.

14. Gould MK, Dembitzer AD, Doyle RL, Hastie TJ and Garber AM: Low-molecular-weight heparins compared with unfractionated heparin for treatment of acute deep venous thrombosis. A meta-analysis of randomized, controlled trials. Ann Intern Med 130: 800-809, 1999.

15. Kakkar VV, Cohen AT, Edmonson RA, Phillips MJ, Cooper DJ, Das SK, Maher KT, Sanderson RM, Ward VP and Kakkar S; The Thromboprophylaxis Collaborative Group: Low molecular weight versus standard heparin for prevention of venous thromboembolism after major abdominal surgery. Lancet 341: 259-265, 1993.

16. Vedantham S, Goldhaber SZ and Kahn SR, Julian J, Magnuson E, Jaff MR, Murphy TP, Cohen DJ, Comerota AJ, Gornik HL, et al: Rationale and design of the ATTRACT Study: A multicenter randomized trial to evaluate pharmacomechanical catheter-directed thrombolysis for the prevention of postthrombotic syndrome in patients with proximal deep vein thrombosis. Am Heart J 165: 523-530.e3, 2013. 
17. Bækgaard N, Klitfod L and Broholm R: Safety and efficacy of catheter-directed thrombolysis. Phlebology 27 (Suppl 1): 149-154, 2012.

18. Nazir SA, Ganeshan A, Nazir S, and Uberoi R: Endovascular treatment options in the management of lower limb deep venous thrombosis. Cardiovasc Intervent Radiol 32: 861-876, 2009.

19. Guyatt GH, Akl EA, Crowther M, Gutterman DD and Schuünemann HJ; American College of Chest Physicians Antithrombotic Therapy and Prevention of Thrombosis Panel: Executive summary: Antithrombotic therapy and prevention of thrombosis, 9th ed: American College of Chest Physicians Evidence-Based Clinical Practice Guidelines. Chest 141: 7S-47S, 2012.

20. Gao B, Zhang J, Wu X, Han Z, Zhou H, Dong D and Jin X: Catheter-directed thrombolysis with a continuous infusion of low-dose urokinase for non-acute deep venous thrombosis of the lower extremity. Korean J Radiol 12: 97-106, 2011.

21. Baker R, Samuels S, Benenati JF, Powell A and Uthoff $\mathrm{H}$ Ultrasound-accelerated vs standard catheter-directed thrombolysis - a comparative study in patients with iliofemoral deep vein thrombosis. J Vasc Interv Radiol 23: 1460-1466, 2012.

22. Zhang X, Ren Q, Jiang X, Sun J, Gong J, Tang B and Chen Y: A prospective randomized trial of catheter-directed thrombolysis with additional balloon dilatation for iliofemoral deep venous thrombosis: A single center experience. Cardiovasc Intervent Radiol 37: 958-968, 2014.
23. Lin PH, Zhou W, Dardik A, Mussa F, Kougias P, Hedayati N, Naoum JJ, El Sayed H, Peden EK and Huynh TT: Catheter-direct thrombolysis versus pharmacomechanical thrombectomy for treatment of symptomatic lower extremity deep venous thrombosis. Am J Surg 192: 782-788, 2006

24. Kuo WT: Optimizing catheter-directed thrombolysis for acute deep vein thrombosis: Validating the open vein hypothesis. J Vasc Interv Radiol 24: 24-26, 2013

25. Baekgaard N, Broholm R, Just S, Jørgensen M and Jensen LP: Long-term results using catheter-directed thrombolysis in 103 lower limbs with acute iliofemoral venous thrombosis. Eur J Vasc Endovasc Surg 39: 112-117, 2010.

26. Protack CD, Bakken AM, Patel N, Saad WE, Waldman DL and Davies MG: Long-term outcomes of catheter directed thrombolysis for lower extremity deep venous thrombosis without prophylactic inferior vena cava filter placement. J Vasc Surg 45: 992-997, 2007.

27. Herrera S and Comerota AJ: Embolization during treatment of deep venous thrombosis: Incidence, importance, and prevention. Tech Vasc Interv Radiol 14: 58-64, 2011.

28. Matsuda A, Yamada N, Ogihara Y, Tsuji A, Ota S, Ishikura K, Nakamura $\mathbf{M}$ and Ito $\mathbf{M}$ : Early and long-term outcomes of venous stent implantation for iliac venous stenosis after catheter-directed thrombolysis for acute deep vein thrombosis. Circ J 78: 1234-1239, 2014 